\title{
Recovering Spectral Data from Natural Scenes with an RGB Digital Camera and Colored Filters
}

\section{Eva M. Valero, ${ }^{1 *}$ Juan L. Nieves, ${ }^{1}$ Sérgio M. C. Nascimento, ${ }^{2}$ Kinjiro Amano, ${ }^{3}$ David H. Foster ${ }^{3}$}

\footnotetext{
${ }^{1}$ Departamento de Óptica, Facultad de Ciencias, Universidad de Granada, 18071 Granada, Spain

${ }^{2}$ Department of Physics, Minho University, Campus de Gualtar, 4710-057 Braga, Portugal

${ }^{3}$ Sensing, Imaging, and Signal Processing Group, School of Electrical and Electronic Engineering, University of Manchester, Manchester M60 1QD, United Kingdom
}

Received 26 June 2006; revised 26 September 2006; accepted 25 October 2006

\begin{abstract}
Many spectral-recovery methods using RGB digital cameras assume the underlying smoothness of illuminant and reflectance spectra, and apply low-dimensional linear models. The aim of the present work was to test whether a direct-mapping method could be used instead of a linear-models approach to recover spectral radiances and reflectances from natural scenes with an $R G B$ digital camera and colored filters. In computer simulations, a conventional RGB digital camera with up to three colored filters was used to image scenes drawn from a hyperspectral image database. Three measures were used to evaluate recovery with the direct-mapping method: goodness-of-fit, root-mean-square error, and a color-difference metric. It was found that with two and three filters both spectral radiances and reflectances could be recovered sufficiently accurately for many practical applications. With little increase in computational complexity, an RGB camera and a few colored filters can provide significantly better recovery of natural scenes than an RGB camera alone. (C) 2007 Wiley Periodicals, Inc. Col Res Appl, 32, 352-360, 2007; Published online in Wiley InterScience (www.interscience.wiley.com). DOI $10.1002 / \mathrm{col} .20339$
\end{abstract}

\footnotetext{
*Correspondence to: Eva M. Valero (e-mail: valerob@ugr.es).

Contract grant sponsor: Spanish Ministry of Education and Science, Contract grant number: DPI2004-03734; Contract grant sponsor: Engineering and Physical Sciences Research Council, Contract grant numbers: GR/R39412/01, EP/B000257/1; Contract grant sponsor: Fundação para a Ciência e Tecnologia, Contract grant number: POSI/SRI/40212/ 2001.
}

(C) 2007 Wiley Periodicals, Inc.
Key words: reflectance; multispectral imaging systems; illuminants; natural scenes

\section{INTRODUCTION}

The goal of multispectral imaging is to recover radiance or reflectance spectra at each pixel in a scene of interest. $^{1-4}$ Typically, a multispectral system consists of a digital camera coupled to a range of spectrally broad-band or narrow-band filters. If the number of filters is sufficiently large and their bandwidths are sufficiently small, as with a hyperspectral imaging system, ${ }^{2,5,6}$ spectral data can be recovered exactly. ${ }^{5-9}$ But with just a few broad-band filters, spectral recovery presents an ill-posed problem.

Many multispectral-imaging methods exploit the underlying smoothness of signal spectra, ${ }^{10}$ with illuminants ${ }^{11-13}$ and spectral reflectances ${ }^{14,15}$ represented by low-dimensional models based on principal component analysis (PCA) or independent component analysis (ICA). ${ }^{16-20}$ Thus, given a linear model, ${ }^{10,21}$ if the number of PCA or ICA coefficients of a particular set of spectra is the same as the number of camera responses (three in the simplest trichromatic case), then the spectra can be derived by an inverse transformation of the set of camera responses, with the forward transformation being estimated from a representative ("training") data set. If the number of coefficients is more than the number of response values, then the latter may need to be increased by imaging the scene under different illuminants or by introducing colored fil- 
ters one at a time in front of the camera to modify the sensor spectra. ${ }^{18,22,23}$

Rather than an initial PCA or ICA being performed, however, the set of signal spectra may instead be estimated directly from the set of camera responses. These responses may be obtained from the camera itself or, for the present purposes, calculated from a set of known camera spectral sensitivities (Camera Spectral Sensitivities and Colored Filters section). For a conventional RGB digital camera, the set of camera responses comprises a matrix of three values for each pixel over all the pixels in the scene. If colored filters are introduced, then the set of camera responses comprises several of these matrices (Computations section). The "direct-mapping" method ${ }^{24}$ is described in more detail in the next section.

\section{Direct Mapping of Camera Responses and Spectra}

Given a set of training spectra $\mathbf{S}$ (which can be spectral radiances or reflectances associated with a certain sample of pixels in the scene) and the corresponding set of camera responses $\mathbf{R}$, a recovery transformation matrix $\mathbf{D}$ is defined by $\mathbf{D}=\mathbf{S R}^{+}$, where $\mathbf{R}^{+}$is the pseudoinverse of $\mathbf{R}$ (this notation differs from that in Ref. 24). If $\mathbf{R}$ has full rank, then $\mathbf{R}^{+}=\left(\mathbf{R}^{\mathrm{t}} \mathbf{R}\right)^{-1} \mathbf{R}^{\mathrm{t}}$, where $\mathbf{R}^{\mathrm{t}}$ is the transpose of $\mathbf{R}$. An estimate $\hat{\mathbf{S}}_{\mathbf{1}}$ of a set of test spectra $\mathbf{S}_{\mathbf{1}}$ may then be obtained from the corresponding set of camera responses $\mathbf{R}_{1}$ by applying the transformation $\mathbf{D}$, that is, $\hat{\mathbf{S}}_{\mathbf{1}}=\mathbf{D R}_{\mathbf{1}}$ (see Refs. 25 and 26). The direct-mapping method has been applied successfully to art imaging ${ }^{22,27}$ and to illuminant estimation, ${ }^{3}$ but it is unclear whether it remains efficient for radiance or reflectance estimation in conditions where the illumination is uncontrolled. Natural scenes, the subject of the present study, represent a special challenge for spectral-recovery methods.

\section{Recovery of Natural Spectra}

The choice of training spectra used to calculate the recovery transformation is critical in determining its performance: if the set is insufficiently representative, then the recovery transformation will be inaccurate; and if it is too similar to the test set, it will lose generality. In practice, different training sets are used for different applications, for example, oil pigments for multispectral analysis of artworks. ${ }^{22}$ Although a standard in some laboratory applications, the Munsell $\operatorname{set}^{28}$ and the Macbeth ColorChecker, ${ }^{29}$ which encompass some reflectance spectra found in natural environments, seem unlikely to provide optimal training sets because they do not represent the distribution of natural spectral reflectances.

The aim of the present work was to test whether the direct-mapping method could be used instead of a linearmodels approach to recover spectral radiances and reflectances satisfactorily from natural scenes. The natural spectra were obtained by hyperspectral imaging 5 and the recovery matrix was estimated using the simulated res- ponses of a conventional RGB digital camera coupled to several combinations of colored filters.

It was found that natural spectra could indeed be accurately recovered with this simple system, and, as anticipated, accuracy was significantly better when natural radiances and reflectances were used as the training set rather than those from a color chart.

\section{METHODS}

\section{Hyperspectral Data}

Hyperspectral data from thirty natural scenes, fifteen of rural environments and fifteen of urban environments, were drawn from a high-spatial-resolution database. ${ }^{5}$ The scenes were taken from the Minho region of Portugal, which has a temperate climate and variety of vegetation and natural rock formations. The hyperspectral imaging system used a Peltier-cooled digital monochromatic camera with spatial resolution $1344 \times 1024$ pixels (Hamamatsu, model C4742-95-12ER, Hamamatsu Photonics K.K., Japan) with a fast-tunable liquid-crystal filter (VariSpec, model VS-VIS2-10-HC-35-SQ, Cambridge Research \& Instrumentation Inc., Woburn, MA) mounted in front of the lens, with infra-red blocking filter. For each scene, 33 images were captured at 10 -nm intervals over $400-720 \mathrm{~nm}$, with 12-bit intensity resolution at each pixel. These raw image sequences were corrected for noise, stray light, off-axis vignetting, and chromatic differences of magnification or translation. The spectral reflectance at each pixel was estimated by normalizing the corrected signal against that obtained from a neutral reference consisting of a small planar gray (Munsell N5 or N7) surface placed in the scene. The reflected spectral radiance at the reference was measured with a telespectroradiometer (SpectraColorimeter, Model PR-650, Photo Research, Chatsworth, CA), whose calibration was traceable to the National Physical Laboratory (U.K.). The reflected spectral radiance at each pixel was estimated by multiplying the estimated spectral reflectance by the effective incident spectral irradiance at the neutral reference (in turn the quotient of reflected radiance by the known reflectance of the reference). An analysis of the assumptions underlying this estimation procedure for directly and indirectly illuminated surfaces is given in Appendix A of Ref. 30. The method applied here is neutral with respect to the nature of the signal being modeled: both radiance and reflectance spectra were considered.

\section{Camera Spectral Sensitivities and Colored Filters}

The calculated camera responses were obtained using the spectral sensitivities of a digital RGB camera with spatial resolution $1280 \times 1024$ pixels (QImaging, model Retiga 1300, QImaging Corp., Canada) with 12-bit intensity resolution per channel at each pixel. Figure 1(a) shows the spectral sensitivities of the red, green, and blue CCD sensors of the camera. The peak sensitivities and bandwidths 
(a)

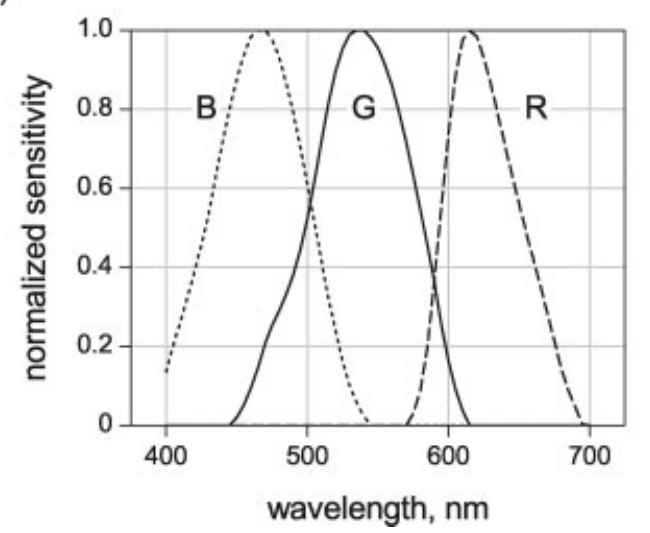

(b)

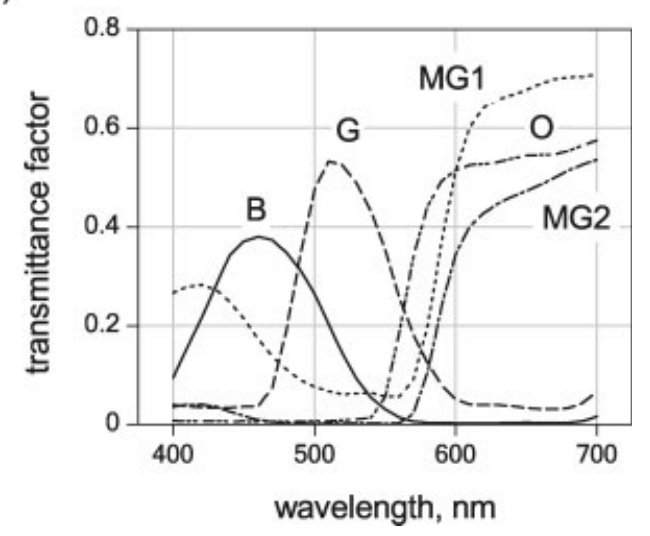

FIG. 1. (a) Normalized spectral sensitivities of RGB digital camera sensors (QImaging Retiga 1300). (b) Spectral transmittances of colored filters used with camera.

of the three channels are similar to those of commercially available digital RGB cameras. ${ }^{31}$ Figure 1(b) shows the spectral transmittances of five colored filters, which, in the simulation, were placed in front of the camera to modify the camera responses. The filters were samples of common transparent acrylic plastic materials: two magenta (MG1 and MG2), one orange (O), one green $(\mathrm{G})$, and one blue (B). This selection was based on a previous exploratory computational optimization of different filters with Gaussian spectral transmittances and variable bandwidths. ${ }^{3}$

\section{Computations}

Different sets of scene fragments were used for the training and test sets. The matrix-training set, used to obtain the recovery matrix, was formed from 30 different fragments, one from each scene, each fragment of size $151 \times 151$ pixels. The following three additional data sets were used for verification of the method's accuracy. Test set 1 was formed from three randomly selected fragments of the matrix-training set. Test set 2 was formed from 30 fragments of $61 \times 61$ pixels with none of the pixels in common with those of the matrix training set. And test set 3 was formed by three additional fragments of $151 \times$ 151 pixels from other scenes (close-up views not used in the matrix-training set). The influence of any possible correlation between adjacent pixels was tested for by sampling half of the scenes over alternate pixels vertically and horizontally (i.e., every fourth pixel) and comparing the recovered radiance spectra with those obtained from the unsampled scenes. The two sets of recovered signals (whose distributions were nonnormal by the KolmogorovSmirnov test) were significantly different for all filter combinations $(P<0.001$, Wilcoxon signed rank tests $)$. Since reflectances differed sufficiently over adjacent pixels, introducing larger pixel spacings, which would have lost the spatial integrity of the sample, was considered unnecessary.

The matrix-training set comprised $151 \times 151 \times 30$ (684030) spectra each defined over 400-700 $\mathrm{nm}$. These and other spectra were interpolated at 5-nm intervals to match the sampling interval for the camera spectral sensitivities (originally sampled at 10 -nm intervals), thus yielding a matrix $\mathbf{S}$ of $684030 \times 61$ values. The camera responses $r_{i}$ to these spectra $(i=1,2$, and 3 for red, green, and blue sensors, respectively) were calculated as follows. At each pixel, suppose that $S(\lambda)$ is the signal spectrum; $R_{i}(\lambda)$ is the camera spectral sensitivity for each sensor $i$; and $F(\lambda)$ is the spectral transmittance of one of the selected filters, where $\lambda=400,405, \ldots, 700 \mathrm{~nm}$. Then

$$
r_{i}=\sum_{\lambda=400}^{700} S(\lambda) F(\lambda) R_{i}(\lambda)
$$

With a total of $n$ colored filters, there are $3 n+3$ camera responses for each pixel (including responses with no colored filters) forming the response matrix $\mathbf{R}$ for the entire scene fragment. As indicated in the Introduction, the $(3 n+3) \times 61$ recovery matrix $\mathbf{D}$ was calculated from the pseudoinverse $\mathbf{R}^{+}$of $\mathbf{R}$ by

$$
\mathbf{D}=\mathbf{S R}^{+} \text {. }
$$

The matrix $\mathbf{D}$ was calculated for the camera without any colored filter, with one filter (each one of the set of five), with two filters (four different combinations of two colored filters), and with three filters (two different combinations). The filters used in the two-filter combinations were selected from those yielding the best performance with one-filter recovery. In turn, the filters used in the three-filter combinations were selected from those yielding the best performance with two-filter recovery. Four different two-filter combinations and two different three-filter combination were tested.

\section{Evaluation of Spectral and Colorimetric Quality of Recovered Signals}

Three measures of recovery performance were used to avoid the idiosyncrasies of any single measure ${ }^{32,33}$ : two were spectral measures and one was colorimetric, taking 
(a)

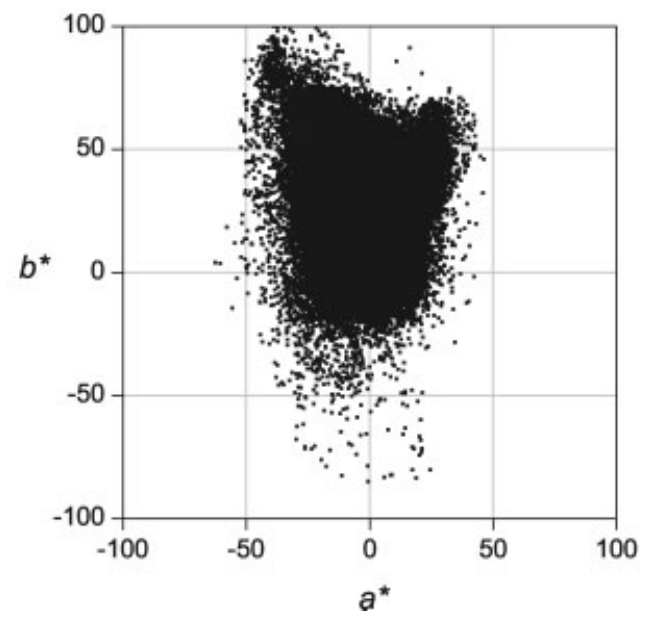

(b)

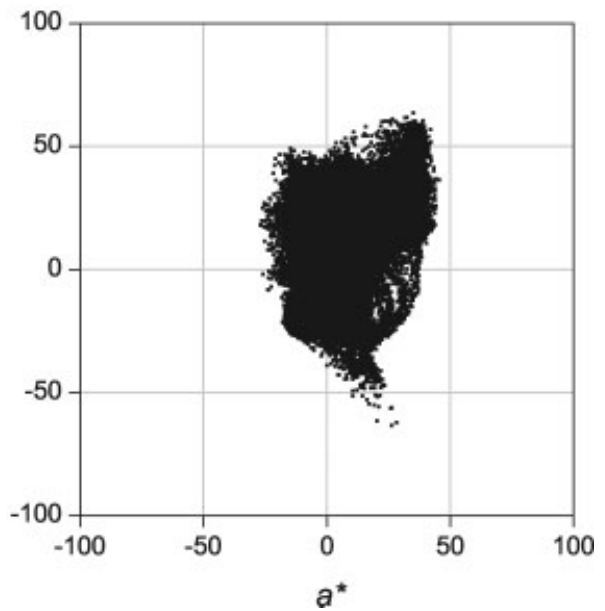

FIG. 2. CIELAB plot of $\left(a^{*}, b^{*}\right)$ values of the matrix-training set for (a) rural data and (b) urban data.

into account the eye's sensitivity to color differences. The first was the goodness-of-fit coefficient (GFC), defined as the cosine of the angle between the recovered signal $\hat{S}$ and original signal $S$, thus

$$
\mathrm{GFC}=\frac{\sum_{\lambda=400}^{700} \widehat{S}(\lambda) S(\lambda)}{\left(\sum_{\lambda=400}^{700} \widehat{S}(\lambda)^{2}\right)^{1 / 2}\left(\sum_{\lambda=400}^{700} S(\lambda)^{2}\right)^{1 / 2}} .
$$

This commonly used measure of spectral similarity has the advantage of not being affected by scale factors. Values range from 0 to $100 \%$, with $\mathrm{GFC} \geq 99.5 \%$ corresponding to acceptable recovery and GFC $\geq 99.99 \%$ to an almost-exact fit. ${ }^{32,34}$ The second measure was the rootmean-square error (RMSE), which, for radiances, was scaled by the maximum of each over the wavelength range. The third was the CIELAB color difference $\Delta E_{\mathrm{ab}}^{*}$, which was calculated with reference to the color signal of a white patch included in the scene for illuminant estimation. For reflectances, an equienergy illuminant was assumed for the evaluation of color differences. Real or standard illuminants were not used since the main aim was to compare different filter combinations. This equienergy illuminant should not be confused with the scene illuminant recorded at the time of image acquisition (Hyperspectral Data section). These three measures were applied to the spectral recovery of test sets 1,2 , and 3 .

\section{RESULTS AND COMMENT}

From the direct-mapping method, two estimates of the recovery matrix D were obtained. The first related camera responses to spectral radiances and the second related camera responses to spectral reflectances. By definition, the spectral radiance at a pixel coincides with the product of the spectral reflectance and the spectral power distribution of the scene illuminant (Hyperspectral Data section), but the recovery of the two kinds of estimates was not identical. Radiance and reflectance estimates are therefore considered separately in the following two subsections (Spectral-Radiance Recovery and Spectral-Reflectance Recovery sections), respectively.

Recovery also depended on the matrix-training data. Although in CIELAB space the rural and urban matrixtraining data had similar lightness ranges, the distribution of their chromaticities differed, as the plots of $b^{*}$ versus $a^{*}$ values in Fig. 2 show. The differences between the two sets of data are considered in Differences Between Rural and Urban Scenes section.

\section{Spectral-Radiance Recovery}

Figure 3 shows four examples of spectral-radiance recovery for different scene fragments. The examples on the top row, (a) and (b), are from test set 1 and those on the bottom row, (c) and (d), are from test set 2. Those on the left, (a) and (c), have GFC and RMSE values in the top 5th centile and those on the right, (b) and (d), in the bottom 5th centile. The large differences in radiance scales are due to some recovered radiances being from areas directly illuminated by the sun and others from areas in shadow. ${ }^{30}$ Recovery appears to be independent of signal scale, owing to the linearity of the camera responses and the direct-mapping method.

Recovery clearly improves as the number of camera filters increases. The filter combinations shown correspond to those producing the best results with the test sets used, although the worst two-filter combination was still better than any filter alone, and the worst three-filter combination was still better than any two-filter combination. But introducing more colored filters, with a consequent increase in image-acquisition time, was thought unlikely to produce significant improvement.

Table I shows the mean (and sample standard deviation) of GFC, RMSE, and $\Delta E_{\mathrm{ab}}^{*}$ values calculated across all spectra (68403 for test sets 1 and 3, 111630 for test set 2). Only the best filter combination is shown for two- 
(a)

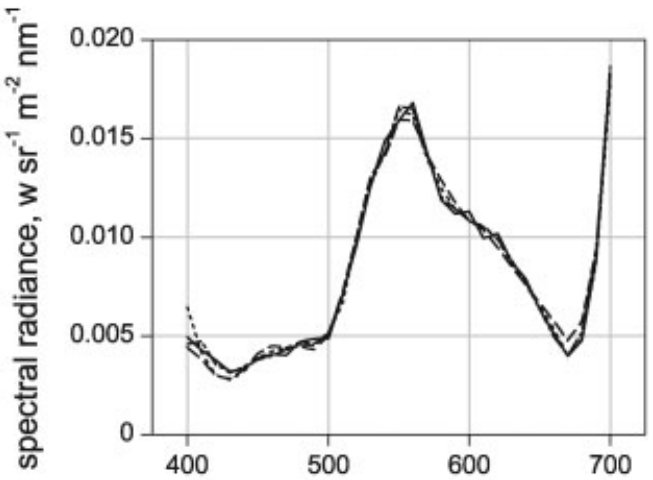

(c)

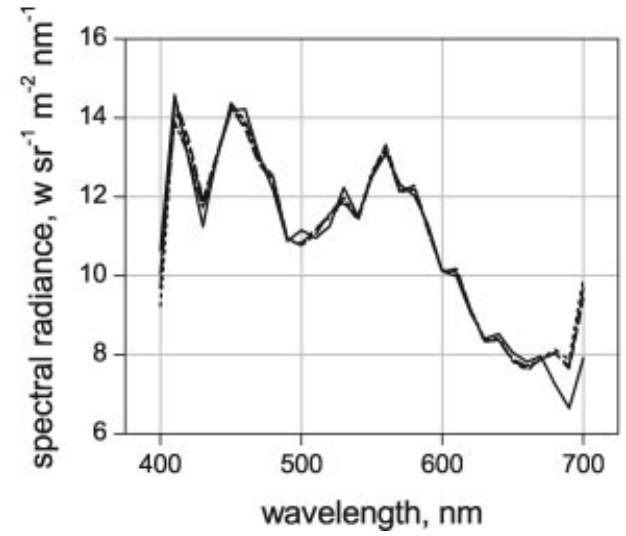

(b)

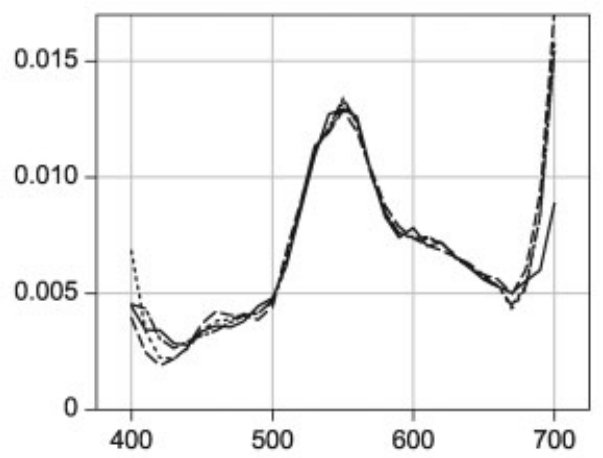

- original

----- G

........ $\mathrm{G}+\mathrm{O}$ $\mathrm{G}+\mathrm{O}+\mathrm{MG} 2$

(d)

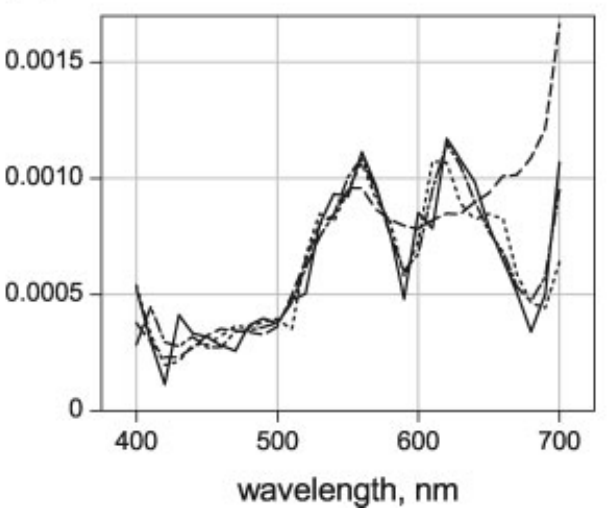

FIG. 3. Recovery of spectral radiances for two members (a) and (b) of test set 1 and for two members (c) and (d) of test set 2 with (a) and (c) having goodness-of-fit coefficient (GFC) in top 5th centile, and (b) and (d) in bottom 5th centile. GFC values for color filter $\mathrm{G}$ were (a) $99.87 \%$, (b) $98.17 \%$, (c) $99.93 \%$, and (d) $94.62 \%$.

and three-filter combinations. The RMSE values are reasonably low particularly for two- and three-filter combinations. Color differences $\Delta E_{\mathrm{ab}}^{*}$ do not exceed unity, implying that there would be little noticeable colorimetric difference between recovered and original signals. The effects of number of filters and of filter type were confirmed by a nonparametric Friedman's two-way ANOVA applied to the three measures, the results of which were statistically very highly significant $(P<0.001)$. These statistical tests show that the differences in recovery with one, two and three filters are significant and that recovery with the best two- and three-filter combinations is significantly better than with other possible combinations.

Recovery was better for test set 1 than for test sets 2 and 3. This was not unexpected, since estimation of the recovery matrix was based on test set 1 . Figure 4 shows visualized images of a fragment of scene radiance spectra (a) and its recovery (b) with three colored filters. The visualization was based on the calculated camera response values. Colorimetric differences between the recovered and original images are not visually detectable, as predicted by the $\Delta E_{\mathrm{ab}}^{*}$ values, which were less than unity.

TABLE I. Quality of recovery of spectral radiances from natural scenes.

\begin{tabular}{|c|c|c|c|c|c|c|c|c|c|}
\hline \multirow[b]{2}{*}{ Measure } & \multirow[b]{2}{*}{ Test set } & \multicolumn{8}{|c|}{ Color filters } \\
\hline & & None & MG 1 & MG 2 & B & $\mathrm{G}$ & O & $\mathrm{G}+\mathrm{O}$ & $\mathrm{G}+\mathrm{O}+\mathrm{MG} 2$ \\
\hline \multirow[t]{3}{*}{ GFC (\%) } & 1 & $99.20(0.76)$ & $99.64(0.57)$ & $99.67(0.56)$ & $99.55(0.43)$ & $99.50(0.57)$ & $99.62(0.41)$ & $99.71(0.34)$ & $99.86(0.12)$ \\
\hline & 2 & $97.06(7.14)$ & $98.34(4.88)$ & 98.46 (4.52) & 98.54 (3.46) & $98.08(5.05)$ & 98.46 (3.54) & 99.01 (2.39) & $99.34(1.58)$ \\
\hline & 3 & 97.05 (5.61) & 98.64 (3.44) & 98.69 (2.92) & $98.53(3.06)$ & 98.61 (3.26) & $98.61(2.24)$ & $98.96(1.80)$ & $99.26(1.40)$ \\
\hline \multirow[t]{3}{*}{ RMSE (\%) } & 1 & $8.28(2.46)$ & $5.12(2.09)$ & $4.83(2.11)$ & 5.99 (1.92) & $6.42(1.84)$ & $5.53(1.48)$ & 4.72 (1.82) & $3.32(1.15)$ \\
\hline & 2 & $9.96(3.96)$ & $6.65(3.48)$ & $6.44(3.29)$ & $6.82(3.14)$ & 7.37 (3.67) & $7.02(3.15)$ & 5.65 (3.04) & $4.55(2.83)$ \\
\hline & 3 & 9.31 (3.35) & $6.34(2.50)$ & $6.17(2.50)$ & $6.73(2.54)$ & $6.37(2.54)$ & $6.21(2.82)$ & $6.14(2.24)$ & $5.00(2.17)$ \\
\hline \multirow[t]{3}{*}{$\Delta E_{\mathrm{ab}}^{\star}$} & 1 & $0.32(0.16)$ & $0.09(0.05)$ & $0.10(0.06)$ & $0.22(0.13)$ & $0.06(0.04)$ & $0.18(0.12)$ & $0.09(0.06)$ & $0.05(0.04)$ \\
\hline & 2 & $0.63(0.61)$ & $0.24(0.28)$ & $0.28(0.32)$ & $0.35(0.32)$ & $0.17(0.20)$ & $0.35(0.46)$ & $0.12(0.14)$ & $0.09(0.12)$ \\
\hline & 3 & $1.01(0.76)$ & $0.28(0.18)$ & $0.36(0.26)$ & $0.24(0.12)$ & $0.15(0.09)$ & $0.43(0.32)$ & $0.08(0.06)$ & $0.08(0.06)$ \\
\hline
\end{tabular}

Mean (sample SD) over 68403 spectra with test sets 1 and 3 and 111630 spectra with test set 2 for three measures of performance and eight filter conditions. 


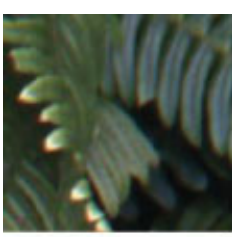

(a)

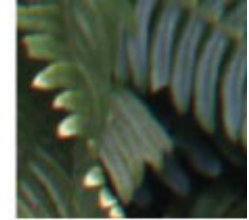

(b)
FIG. 4. Visualized image of (a) original scene fragment and (b) fragment recovered with three colored filters.

\section{Spectral-Reflectance Recovery}

Figure 5 shows four examples of spectral-reflectance recovery for different scene fragments. The examples on the top row, (a) and (b), are from test set 1 and those on the bottom row, (c) and (d), are from test set 2 . Those on the left, (a) and (c), have GFC and RMSE values in the top 5th centile and those on the right, (b) and (d), in the bottom 5 th centile.

Table II shows the mean (and sample standard deviation) of GFC, RMSE, and $\Delta E_{\mathrm{ab}}^{*}$ values calculated across all spectra (68403 for test sets 1 and 3, 111630 for test set 2). Again, only the best filter combination is shown for two- and three-filter combinations. As with the analysis of spectral-radiance recovery, the strong effects of number of filters and of filter type were confirmed by a nonparametric Friedman's two-way ANOVA applied to the three measures, the results of which were very highly significant $(P<0.001)$.

This level of performance is compatible with that found in other studies using the direct-mapping method or other methods for recovering spectral data from paints and photographic standards under controlled illumination. ${ }^{10,19,35}$

\section{Differences Between Rural and Urban Scenes}

In the foregoing analyses, spectra from rural and urban scenes were unseparated. But because the two kinds of spectra have different physical origins and characteris$\operatorname{tics}^{36}$ (Fig. 2), it is possible that the effectiveness of the recovery might also differ. Accordingly, recovery matrices were estimated afresh for both radiance and reflectance spectra after separating rural and urban scenes from test sets 1 and 2 . Test set 3 was used as a reference test for both matrices, so that the two recovery matrices could then be compared for the same set of radiance and reflectance spectra. For test set 1, recovery of radiances was slightly better with rural scenes with the rural recovery matrix than for urban scenes with the urban recovery matrix (rural recovery with the $\mathrm{G}$ filter yielded a mean GFC of $99.64 \%$, RMSE of $5.35 \%$, and $\Delta E_{\mathrm{ab}}^{*}$ of 0.04 , whereas urban recovery with the same filter yielded a GFC of $99.66 \%$, RMSE of $6.18 \%$, and $\Delta E_{\mathrm{ab}}^{*}$ of 0.08 ). For test set 2 , recovery of radiances was somewhat better for urban (a)

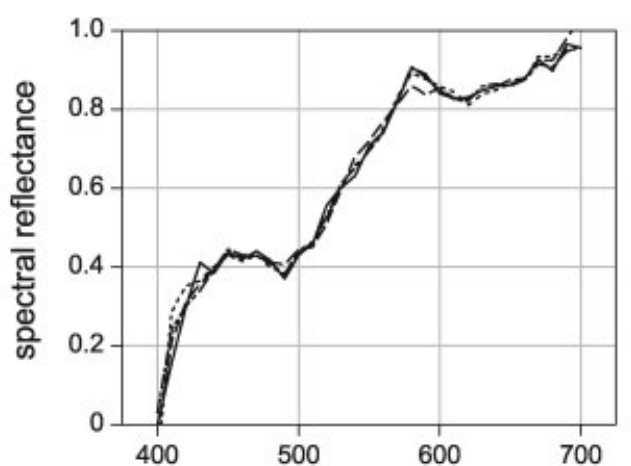

(c)

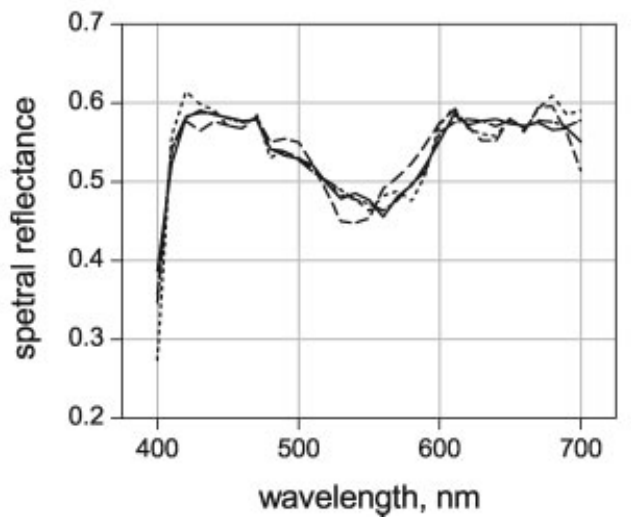

(b)

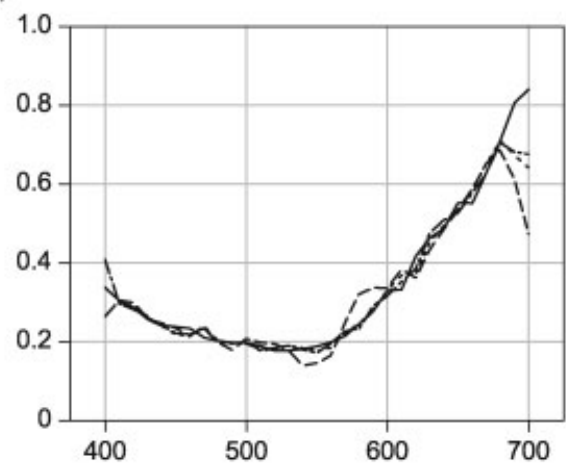

(d)

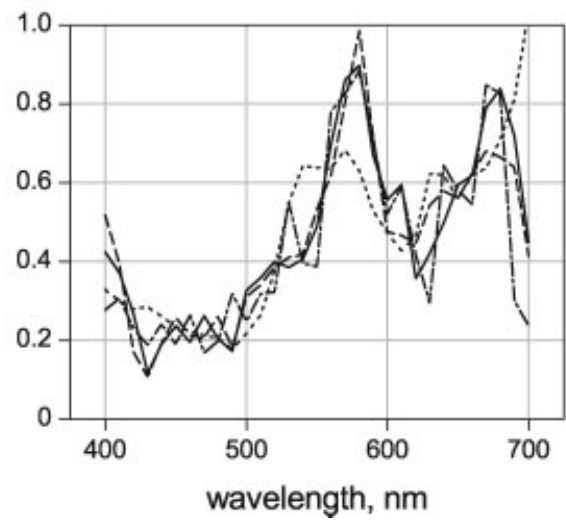

FIG. 5. Recovery of spectral reflectances for two members (a) and (b) of test set 1 and for two members (c) and (d) of test set 2, with (a) and (c) having GFC in top 5th centile and (b) and (d) in bottom 5th centile. GFC values for color filter B were (a) $99.93 \%$, (b) $98.41 \%$, (c) $99.93 \%$, and (d) $93.80 \%$. 
TABLE II. Quality of recovery of spectral reflectances from natural scenes.

\begin{tabular}{|c|c|c|c|c|c|c|c|c|c|}
\hline \multirow[b]{2}{*}{ Measure } & \multirow[b]{2}{*}{ Test set } & \multicolumn{8}{|c|}{ Color filters } \\
\hline & & None & MG 1 & MG 2 & B & $\mathrm{G}$ & O & $\mathrm{G}+\mathrm{O}$ & $\mathrm{G}+\mathrm{O}+\mathrm{MG} 2$ \\
\hline \multirow[t]{3}{*}{ GFC (\%) } & 1 & 99.07 (1.65) & 99.45 (1.09) & $99.49(1.07)$ & $99.63(0.70)$ & 99.35 (1.17) & $99.59(0.69)$ & $99.75(0.42)$ & $99.90(0.10)$ \\
\hline & 2 & $96.45(8.18)$ & $98.00(4.62)$ & $98.13(4.28)$ & $98.63(2.96)$ & $97.64(5.44)$ & $98.39(3.17)$ & 98.99 (2.58) & 99.31 (1.79) \\
\hline & 3 & $96.26(6.30)$ & $97.18(3.87)$ & $97.30(3.75)$ & 98.02 (3.32) & $96.86(4.21)$ & $97.64(3.40)$ & 98.68 (2.40) & 98.96 (1.97) \\
\hline \multirow{3}{*}{ RMSE (\%) } & 1 & $7.24(5.27)$ & $5.49(4.15)$ & $5.20(4.12)$ & $4.51(3.45)$ & $6.03(4.27)$ & 4.88 (3.28) & $3.75(2.77)$ & 2.47 (1.32) \\
\hline & 2 & $5.54(6.11)$ & 3.59 (3.65) & 3.47 (3.57) & 3.17 (3.34) & $3.96(3.96)$ & 3.41 (3.38) & $2.68(2.88)$ & $2.02(2.04)$ \\
\hline & 3 & $2.23(1.40)$ & $2.25(1.25)$ & 2.18 (1.23) & $1.63(1.13)$ & 2.46 (1.35) & 1.99 (1.13) & $1.40(0.92)$ & $1.25(0.80)$ \\
\hline \multirow[t]{3}{*}{$\Delta E_{\mathrm{ab}}^{\star}$} & 1 & $1.03(0.66)$ & $0.53(0.42)$ & $0.54(0.45)$ & $0.50(0.27)$ & $0.43(0.39)$ & $0.57(0.44)$ & $0.24(0.16)$ & $0.18(0.14)$ \\
\hline & 2 & $1.15(0.94)$ & $0.68(0.84)$ & $0.73(0.82)$ & $0.61(0.55)$ & $0.53(0.79)$ & $0.73(0.85)$ & $0.25(0.33)$ & $0.22(0.32)$ \\
\hline & 3 & $1.84(1.25)$ & $1.07(0.74)$ & $1.18(0.82)$ & $0.60(0.55)$ & $0.84(0.63)$ & $1.26(0.96)$ & $0.31(0.33)$ & $0.27(0.31)$ \\
\hline
\end{tabular}

Mean (sample SD) over 68403 spectra with test sets 1 and 3 and 111630 spectra with test set 2 for three measures of performance and eight filter conditions. Color differences $\Delta E_{\mathrm{ab}}^{\star}$ were evaluated for an equienergy illuminant.

scenes with the urban recovery matrix than for rural scenes with the rural recovery matrix (for example, urban recovery with the $\mathrm{G}$ filter yielded a mean GFC of $99.37 \%$, RMSE of $6.82 \%$, and $\Delta E_{\mathrm{ab}}^{*}$ of 0.15 , whereas rural recovery with the same filter yielded a mean GFC of $96.67 \%$, RMSE of $8.22 \%$, and $\Delta E_{\text {ab }}^{*}$ of 0.21 ). Wilcoxon matched-pairs signed rank tests showed that recovery of radiances for rural scenes with the rural recovery matrix was significantly better $(P<0.001)$ than with the general recovery matrix for undifferentiated scenes, and analogously for urban scenes.

When rural and urban recovery matrices were applied to the same set of radiances from test set 3 , recovery was better with the rural recovery matrix (for example, rural recovery with the $G$ filter yielded a mean GFC of $98.70 \%$, RMSE of $5.97 \%$, and $\Delta E_{\mathrm{ab}}^{*}$ of 0.12 , whereas urban recovery with the same filter yielded a mean GFC of $97.44 \%$, RMSE of $9.62 \%$, and $\Delta E_{\mathrm{ab}}^{*}$ of 0.41 ). This is reasonable, since the three close-up scenes could all be classified as rural. Moreover, results with the general recovery matrix applied to test set 3 were worse than with the rural recovery matrix, but better than with the urban recovery matrix (Wilcoxon matched-pairs signed rank tests; $P<0.001)$.

As to the best filter combinations, there were small differences for rural and urban scenes with one filter, but the same trends were found with two- and three-filter combinations. Similar results were obtained for recovery of reflectances.

\section{Performance with the Macbeth ColorChecker}

For comparison, the direct-mapping method was also applied to a standard set of reflectances, the Macbeth ColorChecker Chart (Gretag-Macbeth, X-Rite Inc., Grand Rapids, MI), widely used in camera characterization ${ }^{10,30}$ and spectral recovery. ${ }^{35}$ Five different sets of 240 reflectances from test set 1 were randomly selected, and the performance of the recovery matrix for this set was compared with that for the set of ColorChecker reflectances. If the ColorChecker set was representative of natural reflectances, there should be little difference in performance with the two matrices.

Table III shows mean and standard deviation of the quality measures for the two recovery matrices with four different filter combinations. The natural-reflectance recovery matrix always performed better than the ColorChecker recovery matrix, and the differences were all statistically highly significant $(P<0.001)$ according to Wilcoxon matched-pairs signed rank tests.

An additional comparison was made with 558 randomly selected reflectances from test set 2 . The pattern of performance was maintained for all filter combinations (e.g., with the $\mathrm{B}$ filter, the ColorChecker recovery matrix yielded a mean GFC of $96.40 \%$, a mean RMSE of $4.82 \%$, and mean $\Delta E_{\mathrm{ab}}^{*}$ of 2.07 ; the natural-reflectances recovery matrix yielded a mean GFC of $98.69 \%$, a mean RMSE of $3.15 \%$, and mean $\Delta E_{\mathrm{ab}}^{*}$ of 1.02 ). These differences between recovery for the two sets of training spectra may

TABLE III. Quality of recovery of spectral reflectances with recovery matrices based on natural reflectance spectra and on spectra from Macbeth ColorChecker chart.

\begin{tabular}{|c|c|c|c|c|c|}
\hline \multirow[b]{2}{*}{ Recovery matrix } & \multirow[b]{2}{*}{ Measure } & \multicolumn{4}{|c|}{ Color filters } \\
\hline & & None & $\mathrm{B}$ & $\mathrm{B}+\mathrm{O}$ & $\mathrm{B}+\mathrm{G}+\mathrm{MG} 1$ \\
\hline \multirow[t]{3}{*}{ Natural reflectances } & GFC (\%) & $99.22(6.62)$ & $99.74(0.33)$ & $99.86(0.18)$ & $99.91(0.13)$ \\
\hline & RMSE (\%) & $6.10(4.79)$ & $4.45(3.80)$ & $2.79(1.68)$ & $2.13(1.23)$ \\
\hline & $\Delta E_{\mathrm{ab}}^{\star}$ & $1.47(0.90)$ & $0.75(0.69)$ & $0.24(0.19)$ & $0.09(0.07)$ \\
\hline \multirow{3}{*}{ Macbeth ColorChecker } & GFC (\%) & $98.74(1.90)$ & $98.88(1.55)$ & $99.16(6.16)$ & $99.39(0.80)$ \\
\hline & RMSE (\%) & $7.96(5.27)$ & $7.21(5.44)$ & 6.16 & $5.24(3.90)$ \\
\hline & $\Delta E_{\mathrm{ab}}^{*}$ & $1.61(0.92)$ & $1.46(0.96)$ & $0.62(0.78)$ & $0.20(0.17)$ \\
\hline
\end{tabular}

Mean (sample SD) over 240 spectra for three measures of performance and four filter conditions. 
be a consequence of the fact that the Macbeth ColorChecker chart, although covering a much greater area of color space, has fewer reflectances in the region spanned by natural spectra.

\section{CONCLUSIONS}

Natural scenes with complex variations in spatial structure and uncontrolled illumination present particular problems for recovering radiance and reflectance spectra. The present work has shown, however, that a combination of the direct-mapping method and a conventional RGB digital camera with a limited number of colored filters can provide acceptably accurate estimates, complementing related work on recovering illuminant spectra in natural scenes. $^{3,34,37,38}$

Of the different combinations of colored filters, best performance was obtained with three filters. In terms of the three measures, the recovered signal had a goodnessof-fit coefficient better than $99.0 \%$, a root-mean-square error less than $5.0 \%$, and a CIELAB color difference less than 0.27 .

Using a conventional RGB digital camera in combination with a limited number of colored filters offers a less time-consuming and more economical approach to the multispectral capture of natural and artificial scenes than traditional methods, and the direct-mapping method provides acceptable performance without requiring more computationally expensive optimization procedures for error minimization. This approach allows a larger range of spectra to be sampled in the construction of the recovery matrix than with more constrained reflectance sets such as the Macbeth ColorChecker chart. Yet some data grouping is useful. Although good recovery is possible with undifferentiated training sets from urban and rural scenes, recovery was better for rural scenes with just rural scenes as the training set, and for urban scenes with just urban scenes as the training set.

This work was computational and responses were calculated for a particular RGB digital camera and set of colored filters, but its practical application to other cameras and filters, after basic preprocessing operations such as noise removal and correction of inhomogeneities and response nonlinearities, ${ }^{39}$ should be straightforward.

1. Hardeberg JY. Acquisition and reproduction of colored images: Colorimetric and multispectral approaches. Ph.D. Thesis, Ecole Supérieur Nationale des Telécommunications, 1999; Available at http:// www.dissertation.com/book.php?method=ISBN\&book $=1581121350$.

2. Gat N. Imaging spectroscopy using tunable filters: A review. In: Szu HH, Vetterli M, Campbell WJ, Buss JR, editors. Wavelet Applications VII, Proc. SPIE, Vol.4056. Washington: SPIE; 2000. p 5064.

3. Nieves JL, Valero EM, Nascimento SMC, Hernández-Andrés J, Romero J. Multispectral synthesis of daylight using a commercial digital CCD camera. Appl Opt 2005;44:5696-5703.

4. Connah D, Westland S, Thomson MGA. Recovering spectral information using digital camera systems. Color Technol 2001;117:309312.
5. Foster DH, Nascimento SMC, Amano K. Information limits on neural identification of colored surfaces in natural scenes. Vis Neurosci 2004;21:331-336.

6. Nascimento SMC, Ferreira FP, Foster DH. Statistics of spatial coneexcitation ratios in natural scenes. J Opt Soc Am A Opt 2002;19:1484-1490.

7. Chiao CC, Cronin TW, Osorio D. Color signals in natural scenes: Characteristics of reflectance spectra and effects of natural illuminants. J Opt Soc Am A Opt 2000;17:218-224.

8. Párraga CA, Brelstaff G, Troscianko T, Moorehead IR. Color and luminance information in natural scenes. J Opt Soc Am A 1998; 15:563-569.

9. Ruderman DL, Cronin TW, Chiao CC. Statistics of cone responses to natural images: Implications for visual coding. J Opt Soc Am A 1998; 15:2036-2045.

10. Cheung V, Westland S, Li C, Hardeberg JY, Connah D. Characterization of trichromatic color cameras using a new multispectral imaging technique. J Opt Soc Am A 2005;22:1231-1240.

11. Judd DB, MacAdam DL, Wyszecki G. Spectral distribution of typical daylight as a function of correlated color temperature. J Opt Soc Am 1964;54:1031-1040.

12. Marimont DH, Wandell BA. Linear-models of surface and illuminant spectra. J Opt Soc Am A 1992;9:1905-1913.

13. Romero J, García-Beltrán A, Hernández-Andrés J. Linear bases for representation of natural and artificial illuminants. J Opt Soc Am A 1997;14:1007-1014.

14. Parkkinen JPS, Hallikainen J, Jaaskelainen T. Characteristic spectra of Munsell colors. J Opt Soc Am A Opt Image Sci Vis 1989;6:318322.

15. Kohonen O, Parkkinen J, Jääskeläinen T. Databases for spectral color science. Color Res Appl 2006;31:381-390.

16. Wachtler T, Lee TW, Sejnowski TJ. Chromatic structure of natural scenes. J Opt Soc Am A 2001;18:65-77.

17. Imai FH, Berns, RS. Spectral estimation using trichromatic digital cameras. International Symposium on Multispectral Imaging and Color Reproduction for Digital Archives, Chiba, Japan, 1999; University of Chiba, p 42-49.

18. Toyooka S, Hayasaka N. Two-dimensional spectral analysis using broad-band filters. Opt Commun 1997;137:22-26.

19. Shi M, Healey G. Using reflectance models for color scanner calibration. J Opt Soc Am A 2002;19:645-656.

20. Oxtoby EK, Foster DH, Amano K, Nascimento SMC. How many basis functions are needed to reproduce coloured patterns under illuminant changes? XV European Conference on Visual Perception. Perception 2002;S31:66.

21. Maloney LT. Evaluation of linear models of surface spectral reflectance with small numbers of parameters. J Opt Soc Am A 1986;3:1673-1683

22. Imai FH, Taplin LA, Day DC, Day EA, Berns RS. Imaging at the National Gallery of Art, Washington D.C. Munsell Color Science Lab Technical Report; 2002. Available at http://art-si.org/.

23. D'zmura M. Color constancy: Surface color from changing illumination. J Opt Soc Am A 1992;9:490-493.

24. Zhao Y, Taplin LA, Nezamabadi M, Berns RS. Methods of Spectral Reflectance Reconstruction for A Sinarback 54 Digital Camera. Munsell Color Science Lab Technical Report; 2004. Available at http://art-si.org.

25. Day DC. Filter selection for spectral estimation using a trichromatic camera. 2003. Available at http://www.art-si.org/PDFs/Acquisition/ DCDayMSThesis03.pdf.

26. Pratt WK, Mancill CE. Spectral estimation techniques for the spectral calibration of a color image scanner. Appl Opt 1976;15:73-75.

27. Haneishi H, Hasegawa T, Hosoi A, Yokoyama Y, Tsumura N, Miyake Y. System design for accurately estimating spectral reflectance of art paintings. Appl Opt 2000;39:6621-6632.

28. Munsell Color Corporation. Munsell Book of Color-Matte Finish Collection. Baltimore, MD: Munsell Color Corporation; 1976

29. Gretag-Macbeth ColorChecker DC: X-Rite Inc. Grand Rapids, MI; 2003 
30. Foster DH, Amano K, Nascimento SMC, Foster MJ. Frequency of metamerism in natural scenes. J Opt Soc Am A 2006;23:23592372.

31. Thomson M, Westland S. Colour-imager characterization by parametric fitting of sensor responses. Color Res Appl 2001;26:442-449.

32. Imai FH, Rosen MR, Berns RS. Comparative study of metrics for spectral match quality. First European Conference on Color in Graphics, Imaging and Vision. Poitiers (France) 2002; IS\&T, Springfield, VA, p 492-496.

33. Corbalan M, Millan MS, Yzuel MJ. Color measurement in standard CIELAB coordinates using a 3CCD camera: Correction for the influence of the light source. Opt Eng 2000;39:1470-1476.

34. Chiao CC, Osorio D, Vorobyev M, Cronin TW. Characterization of natural illuminants in forests and the use of digital video data to reconstruct illuminant spectra. J Opt Soc Am A 2000;17:1713-1721.
35. Imai FH, Taplin LA, Day EA. Comparative study of spectral reflectance estimation based on broad-band imaging systems. As part of end-to-end color reproduction from scene to reproduction using MVSI; 2003. Available at http://www.art-si.org/PDFs/Acquisition/ ReportBrdBnd_April_2003.pdf.

36. Nassau K. The Physics and Chemistry of Color. The Fifteen Causes of Color. New York: John Wiley; 1983.

37. Hernández-Andrés J, Nieves JL, Valero EM, Romero J. Spectral daylight recovery using only a few sensors. J Opt Soc Am A 2004;21:13-23.

38. López-Álvarez MA, Hernández-Andrés J, Romero J, Lee RL. Designing a practical system for spectral imaging of skylight. Appl Opt 2005;44:5688-5695.

39. Hardeberg JY, Schmitt F, Brettel H. Multispectral color image capture using a liquid crystal tunable filter. Opt Eng 2002;41:2532-2548.

\section{FORTHCOMING MEETINGS}

\section{Fogra Color Management Symposium}

Join the experts and practitioners who are addressing the most important aspects of color management. On 21st/ 22nd February 2008, Fogra is holding their Color Management Symposium in Munich, Germany. The venue is Hotel Arabella Sheraton Bogenhausen in Munich, Germany. The intended audience is color management experts in industry and science, color management users in the field, and key decision makers for prepress and press technologies.

What does this symposium offer?

- The leading experts in the field of color imaging.

- The achievements of the ICC color management and its future.

- Panel discussions "Future workshop" and tutorials.

- Celebration: The birthday party for the 15 th anniversary of ICC.

- Softproof exhibition.

- Simultaneous German/English interpreting.

The program includes: opening and keynote: ICC color management-yesterday, today, and tomorrow; Session 1: color management basics; Session 2: color management proofing; Session 3: exhibition and demos [softproof], get-together, tutorials, color vision, panel discussion "Future Workshop-what will happen the next 15 years?"; Session 4: RGB-workflow (capture-rendering-proofing); Session 5: color management-specialities; Session 6: color management — outdoor color and requirements.

There also will be a party and social program. Birthday party 15 years ICC with banquet and surprises will be held on the evening of the 21st February 2008. Other activities include the Fogra Institute tour, a guided beer tour through traditional Munich breweries, and a snowboard tour.

Further information-if you have any questions about bookings or participation in the symposium, please do not hesitate to contact Inge Burian on +49 8943182 114 or E-mail her on burian@fogra.org. Andreas Kraushaar is responsible for the symposium. He would be happy to answer any questions about the event program and he may be reached on +4989431 82-335 or by E-mail: kraushaar@fogra.org. Please also pay regard to the area "Events" of Fogra's website: www.fogra.org.

Published online in Wiley InterScience (www.interscience.wiley.com). DOI $10.1002 /$ col.20349 